\title{
A Context-Aware Approach for a Collaborative, Pervasive and Adaptive Digital Storytelling
}

\author{
M. Casillo \\ DII \\ Università di Napoli "Federico II" \\ Napoli, Italy \\ mario.casillo@unina.it
}

\author{
F. Colace, M. De Santo \\ DIIn \\ Università degli Studi di Salerno \\ Fisciano (SA), Italy \\ \{fcolace,desanto\}@unisa.it
}

\author{
S. Lemma, M. Lombardi \\ SIMASLab \\ Università degli Studi di Salerno \\ Fisciano (SA), Italy \\ \{slemma,malombardi\}@unisa.it
}

\begin{abstract}
The paper introduces an approach for the realization of a dynamic and contextual storytelling engine that can allow the dynamic supply of narrative contents, not necessarily predetermined and pertinent to the needs and the dynamic behaviors of the users.

In particular, the system adopts an adaptive and social approach, using a contextual model in order to realize a dynamic digital storytelling approach that makes the visit experience more appealing and immersive. A case study and some experimental results are presented and discussed.
\end{abstract}

\section{Introduction}

"By dint of telling his stories, a man becomes the stories. They continue to live after him, and so he becomes immortal" (from "Big Fish" film).

The mystery contained in each "word" is still one of the most debated issues in the entire history of humanity. From this mystery comes the narration and its ability to persuade, to convert, to enchant [3,24].

With the term 'storytelling', we mean a learning methodology that consists in 'telling' a story to catch the attention of an audience, convey the message that the story wants to tell and stimulate a specific desire in the readers, transmitting events in words, images and sounds [1].

The objectives of storytelling are several and vary according to the context of application: in fact, the disciplinary areas are many and go from the literacy in childhood, to the scholastic education and the museum didactics, passing for other uses such as the company management and the psychoanalysis [26].

If today we can think of using a didactic methodology as the storytelling, it is mainly thanks to two factors: from the one hand, a new way to imagine the education seen from a constructivist perspective, on the other hand the diffusion in the latest decades of new technologies [15].

In this context, we talk about 'digital storytelling', of which there are several definitions, but all turn around the idea to combine the art of telling stories with the variety of digital multimedia services, such as images, audios and videos' [23]: mixture that allows expressing and narrating in a vivid way experiences, situations and considerations.

Digital storytelling exists in many forms and encompasses multiple fields; in particular, there are the following typologies: linear, non-linear, adaptive, social/collaborative, mobile and game.

Linear storytelling is a traditional narrative form where the author chooses a predefined plot with a fixed sequence of events that the protagonist cannot modify in any way. The term 'linear' indicates that any part of the content is seen and/or listened to in the same order all the times it is enjoyed, confirming the fact that the reader is pushed to follow the narrative path without the possibility to influence the plot and/or its end [28].

Non-linear storytelling represents a 'not linear' narration form, that is any corpus of contents structured in a way that all the possible ways to cover are multiple and variable [19]

The main idea is that the advancement of the plot is not fixed a priori, but the development of the story and its realization depend on the interaction and the choices of the audience, who from simple reader or spectator becomes a 'co-author'.

Instead, the adaptive approach is based on an interactive narration that allows intervening and interacting with the process of construction of the story adapting it to the alterations caused by the intervention of the user, with the purpose to keep a certain narrative coherence [12].

The use of media and social media for the sharing of contents and narrative artefacts has considerably transformed even the way in which the stories can be described and told.

'Collaborative' and 'social' storytelling are forms of collective construction of a story where the shared narration becomes a socially interactive means that supports and foments the continuous co-participation of the users [9].

If the use of technology has changed the way to tell the stories, the use of new mobile tools has put the problem on the overlapping of the narratological and the spatial parts. Consequently, the researchers and the developers have been pushed to define new approaches able to integrate the two dimensions in order to make the cultural experience as much as possible immediate and at the same time ubiquitous.

Many projects use the mobile component to realize guided narrative itineraries contextualized to the environment, with an instructive, informative or sometimes simply recreational feature, with active users who move

DOI reference number: 10.18293/DMS2016-033 
inside physical places operating on technological devices [14].

Also the support of geolocation (positioning and localization through integrated GPS) has further improved the use of these mobile tools, allowing a strong reinforcement of the experience offered to the visitor. In fact, it is very current the theme of the creation of geolocated interactive narrations aware of the context, that is, of stories whose contents, organized in a narrative sequence, are strongly anchored to the physical places and to other significant parameters of the environmental context $[6,7,20]$.

Therefore, it is understandable how it is essential to give the citizen adaptive services and contents able to transmit right information in the right contexts [33]. A fundamental contribution to this scenery has come from the increasing diffusion of mobile devices that concretely have created pervasive and context-aware computing approaches [21, 22]. Exactly the context- aware computing seems to be the most important approach to realize an adaptive approach to the distribution of services and contents [32]. The Context Awareness marks the systems capable of perceiving the contexts where they and the user act, and of consequently modifying their behavior and exchanged information. These opportunities offered by the new pervasive technologies have deeply affected the world of the services providers [31].

The service used in a given context, encouraging interaction among the parties, favors the integration of resources, expertise, information and interests (before, during and after use).

The mobile devices are also enriched with multimedia contents (among which audio, video, photo files, etc.) and tools to realize these contents (among which integrated cameras, recording microphones, etc.). This allows creating and exchanging multimedia contents in real time and in any place and, therefore, realizing and sharing digital stories during the itineraries of visit. These characteristics are exploited in all the areas, included those connected to tourism and cultural heritage, in particular in the museums [13, 29]. In fact, they represent places where stories can be told and where several approaches of storytelling are tested [4, 11]: basically, the narration has evolved including digital contents to make the participation of the museums' visitors mainly interactive through a more personal and adaptive storytelling.

Finally, in order to improve the sensation of amusement for the user, it is born a storytelling where the videogames become a more effective learning tool than the traditional teaching methods, where the decisions of the player affect the global story of the play [5].

The objective of this work is to indicate an approach for the realization of a dynamic storytelling engine that can allow the dynamic supply of narrative contents, not necessarily predetermined and pertinent to the needs and the dynamic behaviors of the users.

The adaptability to the context, the social networks and the mobile world represent the pivotal points, in order to give a technological solution to the digital storytelling, which combines, indeed, the adaptive, social and mobile approaches.

The adaptive approach inserts itself in order to enhance the idea of a narrative experience able to dynamically change in relation with the elements of the profile and the context. For this purpose, it has been realized a model for the representation of all possible contexts in order to provide users of contextual services. The fundamental feature of the social component of our system is the sharing of the role of the author among many individuals, each of which, in turn, takes part into the creation of a portion or a segment of the story, but none of them can assume its paternity. Finally, we have chosen to create the system through a mobile application as it proves to be a perfect tool to have in real time all necessary information, facilitating the access to a series of information and conducting an educational role too.

In the following paragraphs, we are going to analyze the main works concerning the digital storytelling. Later, we will describe a scenery as example and introduce the general architecture of the proposed model, presenting in detail each module and phase.

The paper ends with the description of a realized application and its experimentation.

\section{Related Works}

In this section, we are going to show several cases of use of the Digital Storytelling, in order to make the visitors' experience more interactive and engaging, proposing personalized stories using new technologies (geolocation, mobile applications, augmented reality, etc.).

In [8] Young has proposed an interactive narrative architecture that 're-projects' the events of the story, using the planning. In the wake of the work by Young, many adaptive narrative theories have been developed, such as the use of an algorithm of user modeling to predict their actions and the trigger events, in real time; in this way, the character of the user is given by their actions with respect to the context of the story.

An example is Reading Glove [30], system of adaptive interactive narration that uses a smart 'recommender system' and a tabletop display. The reasoning engine of the system guides the users through the story, using three different modalities of recommendation: the casual ones, those based on the content of the story and the recommendations based on the user. Therefore, the recommender system operates like a kind of 'expert narrator', which follows the reader through the narration.

A further example is Framework for adaptive storytelling [10]: the story develops around the notions of interest and preferences of the user. This feedback updates the user model according to their interests through the profiling module. The story is made up of a series of correlated events and the event selection module determines what content is more suitable for the interests of the user in order to select the right event for the progression of the story.

Instead, in [2], the purpose has been to try to create an emphatic adaptive narrator. The system, under development, aims at using the emotional expressions generated by an avatar or a robot in addition to the answers of the listeners who are monitored in real time. Using several devices and sensors, the system points at codifying in runtime the listener's positive and negative emotions and on them modifying the techniques of the narrative discourse. 
Then, it is possible to identify and list a very long series of 'collaborative' and 'social' storytelling that have purposes of pre-schooling, amusement, teaching and fruition of cultural heritage; among these, we would like to remember Casting [25], a software system that supports the audio-based narration of collaborative groups in the creation of non-linear stories. Casting is made up of a client and a web portal. In particular, the client allows the users to create a project team, add audio recordings, connect the audio recordings, select and publish a linear story. The client's users can recover the most up-to-date version of the story and synchronize their modifications in local. Instead, the web portal allows the users to publish the podcasts and discuss, comment, vote and reuse audio-based stories.

Moreover, the user's experience, thanks to the mobility and the territorial and thematic localization, can renew itself using the physical environment like a background and plot on which basis structuring and defining stories that wind along the urban topography [18].

The use of augmented reality is one of the services able to improve in a substantial way the interaction and the active participation of the visitor, bringing them in a more engaging way nearer to the narration of the place, superimposing the actual reality to a virtual one that stimulates the imagination and favors sense connections.

Frequency 1550 [27] is a classic example of role- play in augmented reality, presented by Waag Society and realized to allow the students to get closer to the medieval history of the city of Amsterdam. Thanks to the use of smartphone devices, the visitors move in a hybrid reality that alternates the modern and current city with the faraway one of 1550. Structured as a treasure hunt, the students move inside the city guided by the mobile device looking for the solution of the story.

Another example of cohesion of augmented reality and interactive narration with the use of mobile devices is the project AIRIS [17], developed in 2011, which puts together the concept of game with the visit of the city, calling the visitor to participate into the discovery of the place and its history through fluid and competitive modalities that amplify the curiosity and facilitate the access to knowledge heritage, connecting real space and game space. The user is guided inside a physical space that can be more or less extended: an entire city or even only one part of it, a museum, a room, a castle, etc.

In order to identify a personalized story, suitable for the needs of large masses of visitors and tourists, our work has been aimed at the definition of appropriate models and solutions of fruition that make the visit experience more appealing and immersive. In particular, there is the realization of technological solutions for the modulation of information depending on the users and their characteristics, through techniques of dynamic profiling and solutions for the generation of narrative contents coherent with the experiential mission of the visit.In fact, on the basis of the social behavior of each user, of their own interests and contextual information, it has been realized an ontological model, in order to elaborate the obtained data and propose a personalized story of the user, including a series of information about the place where they are and about the places they can visit.

\section{Motivating example}

In this section, we describe a typical example of use of Digital Storytelling in the field of Cultural Heritage and Tourism in order to understand to the best the main characteristics and purposes of the proposed system.

The idea is to support the tourist in order to give them, in an automatic way, a tailor-made story that contains information about the reached place and suggestions about places to visit according to their own interests, obtained through their own and their friends' likes and tags, taken from the social networks.

Therefore, the system will have to give a personalized story that includes:

- information about the visited place (main characteristics, historical news, etc.);

- specific points of interest and events for the user, filtered for category, with multimedia deepening about each point;

- contextual services for the user;

- experiences lived by other users.

For this, we want to realize a system of dynamic and contextual digital storytelling that is able to collect and elaborate information and social contents about the users giving them a personalized story about the place that they are visiting.

Let us consider now a practical example. Frank is a tourist who arrives for the first time at the harbor of Salerno, in Campania (Italy), to spend some days on holiday. Through the realized application, he will be able to obtain immediately a story about the town he wants to visit, enriched by the tourist attractions of that place. Gathering his geographical position, the system will get from the Web all necessary information and will show them to the user who will not have to worry about finding them manually. After this first phase, Frank will be able to decide to make the login using his own Facebook credentials. The system initially will calculate, according to an analysis of the likes of the same user and of his friends, all the typologies of interest and so will be able to further enrich the story through those resources whose categories correspond to the calculated user's profile. Subsequently, on the basis of an analysis of the tagged places, the system will suggest mainly those places previously visited by his friends, including in the narration their stops too. Finally, on the basis on the user's context, according to his/her current position and global profile, the system will also suggest contextual services.

At this point, Frank, through the ad hoc built story, will be able to know the history of Salerno, the points of the town he is mainly interested in, the surrounding places visited by his friends and the experiences lived by the other users, through a latest analysis that the system will make about the comments that the latter have left on TripAdvisor after their visits. Finally, when Frank, from inside the story, will select a link associated to a point of interest, he will have available multimedia contents and useful tools such as, for example, Google Maps, which will calculate on the map the itinerary to reach it, also on the basis of the means available at that moment for the user. 


\section{System architecture}

In this section, we describe the architecture of the proposed system, which has the purpose to give the user a personalized tailor-made story, related to the place where they are.

The system architecture, shown in figure 1, is composed by three main blocks: an adaptive-socialmobile and context-aware digital storytelling module, a data management module and a knowledge base. It is made up of the adaptive-social-mobile and context- aware digital storytelling module, divided into the search, elaboration and presentation submodules, which correspond respectively to three separate phases, which are fetch, decode and execute. The search submodule has the task of collecting the data online in order to give the elaboration submodule information about the user's current position (for example, data from Wikipedia and comments of the user on TripAdvisor), the resources, services and events that are near them (e.g., a restaurant, a cinema) obtained from social networks and our knowledge base and the Facebook profile of the user and their friends. The elaboration submodule manages all received information and gives the presentation submodule the story about the town related to the user's current position, a recommended list of contextual resources, services and events and places tagged by the user and their friends on Facebook.

Finally, the presentation module has the task of proposing, as a well-structured story, all these personalized, adaptive and contextual information. The obtained final result is presented to the user through a mobile application.

The Knowledge Base is a special type of database for the management of knowledge and information: it is continuously updated by the results obtained through interaction with the application and with the Data Management Module. The Knowledge Base is composed of four components: in particular, "Users", representing all users of the application, "Services", which describes all the services of every possible application context, "Resources", which forms all the points of interest and "Events", which describes all events; and finally the Management Module (MM), used both by the administrators of the app and the users themselves. This module deals with some important issues, including: POIs and events management, where the insertion can be done directly from map, manually or by search of interests; services and comments management, interacting with TripAdvisor, Facebook and Google API.

In the following paragraph, all the submodules and the related phases of the proposed module of adaptivesocial-mobile and context-aware digital storytelling will be analyzed in detail.

\subsection{Adaptive-social-mobile and Context- Aware Digital Storytelling Module}

The adaptive-social-mobile and context-aware digital storytelling module, presented in detail in figure 2, consists of three sequential phases: fetch, where information of interest are collected; decode, which allows their interpretation and elaboration; execute, thanks to which the elaborated and personalized information are proposed.

4.1.1. Fetch phase. The fetch phase is made up of three main operations: the acquisition of news about the current position, the research of the resources, services and events and the acquisition of the profile and of the user's interests.

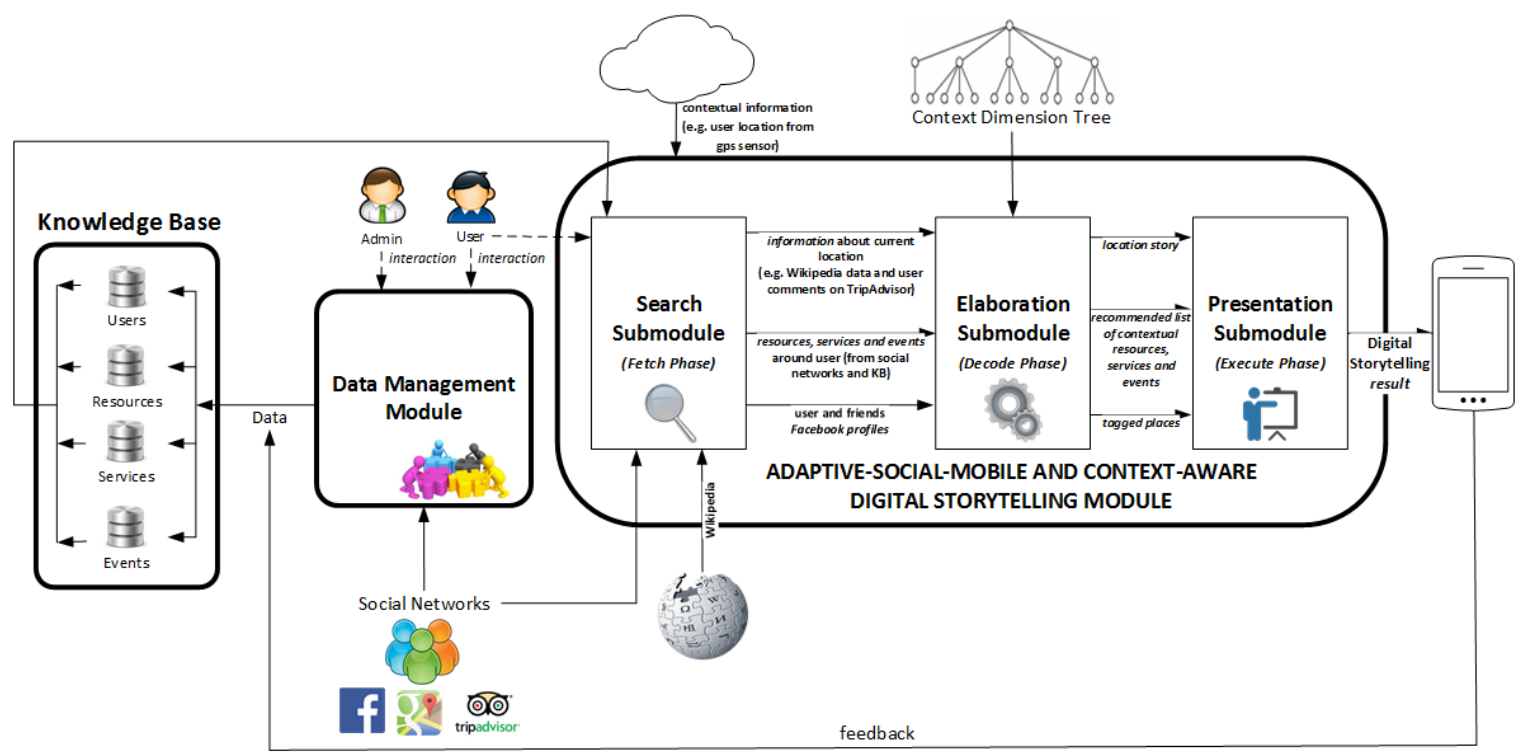

Figure 1. System Architecture 
As regards the first operation, initially it is gathered the position of the user through the GPS sensor of their mobile device: it is necessary to use a service of Reverse Geocoding, that is the process of translation of the geographical coordinates in a 'human-readable' address, made available by the Google Maps API.

Then, to have the description of the locality on the basis of the gathered position, information from Wikipedia have been chosen, in particular using the MediaWiki's RESTful web service API: an easy access to services, data and metadata through HTTP requests.

Often, some coordinates of reference are associated to the pages of Wikipedia and our approach exploits this property: when you make a research of a page related to a village or a city, you obtain the nearest to the user's coordinates.

The story to present is then enriched with the experiences lived by other users, using their comments on TripAdvisor.

Then, it is made the research of the resources from social networks and from our knowledge base. In first case, model uses the map method of the TripAdvisor API, which allows looking for attractions (museums, sports structures, shops, ...), hotels and restaurants through, respectively, the map attractions, map hotels and map restaurant methods. In case the user does not have a social account, they can get the main points of interest of the places where they are using the location-mapper method of the TripAdvisor API. In second case, research is done from our knowledge base, continuously fed by users and results previously obtained from the web.

Finally, for the personalization of information, it is exploited the third operation of the fetch phase of our module, whose objective is to access the social data of the user to get, for example, their profile, their likes, their events and preferred places, their friends: this happens by querying the knowledge base and through the use of the Facebook API and the analysis of some principal methods, such as user likes, user friends, user events and tagged places.

4.1.2. Decode phase. The decode phase is formed by data elaboration and Context Dimension Tree elaboration. The first operation consists in the elaboration of data, in particular in the management of information get from Facebook, in the following correspondence with those of TripAdvisor and in the calculation for the proposition to the user of the resources, that is, of the geographical points of interest according to their own preferences.

In particular, to determine the user's interests, as well as the categories to recommend, we consider their most recent likes on the pages of the Social Network: to any like it corresponds a specific subcategory of Facebook that our system associates to a category of TripAdvisor; for example, to a like on a page related to an art gallery or a painter it will correspond an occurrence of the museum category.

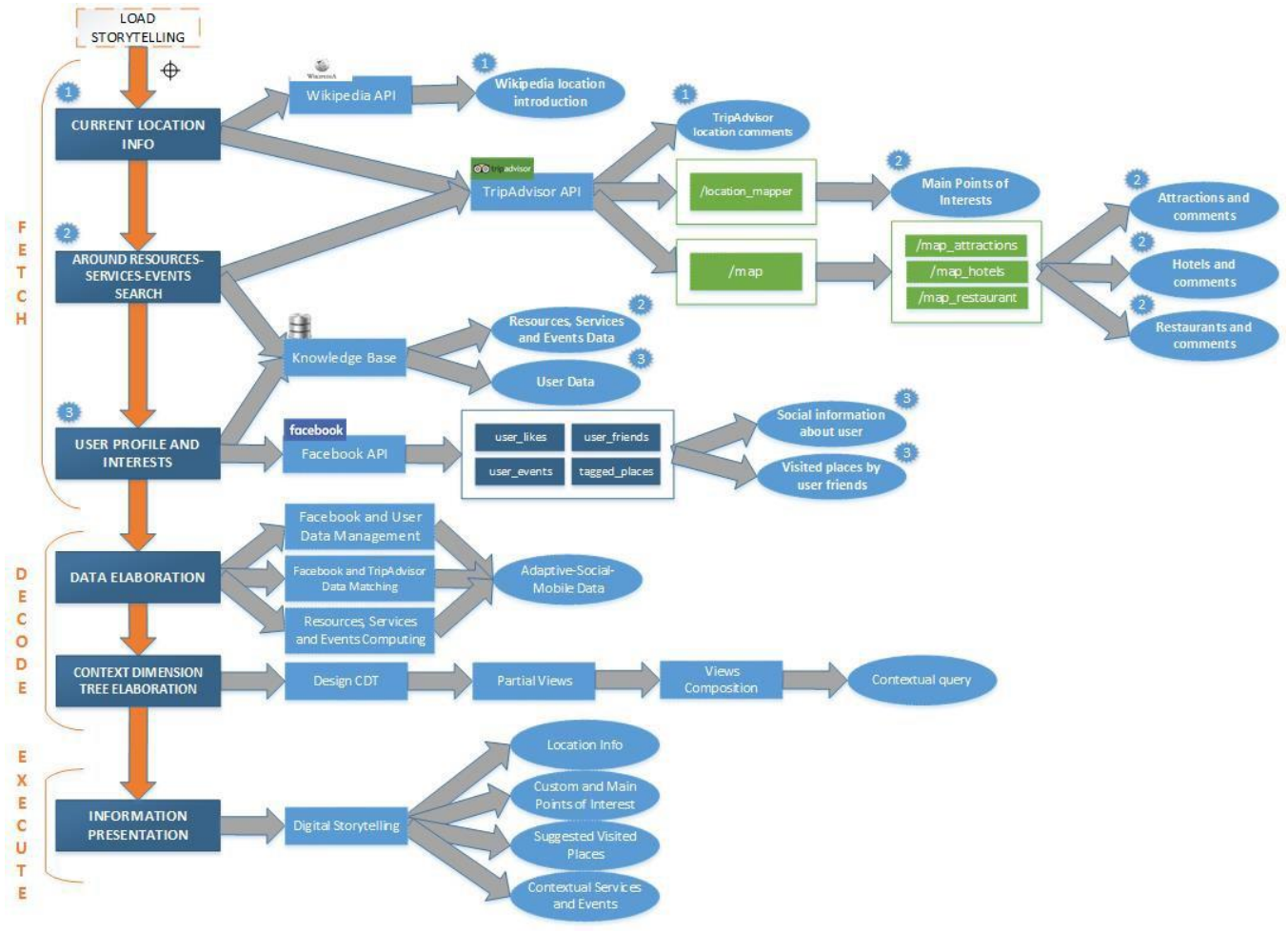

Figure 2. Adaptive-social-mobile and Context-Aware Digital Storytelling 
Through our system, it is possible to calculate the occurrences and get the preferred categories of interest; then, on the basis of the contextual approach, resources, services and events will be proposed to the user, within the personalized story.

In fact, the resources are properly filtered and contextualized based on the second operation of the decode phase: Context Dimension Tree elaboration.

A key element in the design of a contextual application and a Context-Aware System is the representation and management of the context itself: this operation is made through the Context Dimension Tree [16].

CDT is a tree composed of a triad $\langle\mathrm{r}$; N; A $>$ where $\mathrm{r}$ indicates its root, $\mathrm{N}$ is the set of nodes of which it is made of and $\mathrm{A}$ is the set of arcs joining these nodes.

CDT is used to be able to represent, in a graphic form, all possible contexts that you may have within an application.

Nodes present within CDT are divided into two categories, namely dimension nodes and concept nodes. A dimension node, which is graphically represented by the color black, is a node that describes a possible dimension of the application domain; a concept node, on the other hand, is depicted by the color white and represents one of the possible values that a dimension may assume. Each node is identified through its type and a label.

The children of the root node $r$ are all dimension nodes, they are called top dimension and for each of them there may be a sub-tree. Leaf nodes, instead, must be concept nodes. A dimension node can have, as children, only concept nodes and, similarly, a concept node can have, as children, only dimension nodes.

In addition to nodes, you can use other elements: the parameters, which may arise both from a dimension node (graphically represented by a white square) and from a concept node (white triangle), submitting them to particular constraints. In fact, a concept node can have more than one parameter, while a dimension node can have only a parameter and only in case it has not already children nodes. The introduction of parameters is due to their usefulness in shaping the characteristics that can have an infinite or very high number of attributes. For example, a node representing Cost dimension risks having a high number of values that should be specified by as many concept children nodes. In a similar case, it is therefore preferred to use only one parameter, whose value will be specified in each case. Leaf nodes, in addition to concept nodes, can also be parameters.

In general, each node has a parameter corresponding to a domain, $\operatorname{dom}(\mathrm{nP})$. For parameter nodes connected to concept nodes, the domain can be a set of key values from a relational database, while in case of parameter nodes connected to dimension nodes, the domain is a set of possible concept nodes of dimension.

In figure 3, it is shown a general designed CDT, called Meta CDT, which is the starting point for the design of a specific CDT that can be exploited in contextual applications.

You may note six top dimensions, which correspond to the questions of the $5 \mathrm{~W} 1 \mathrm{H}$ method: Location (WHERE), Role (WHO), Time (WHEN), Situation (HOW), Interests (WHAT) and Utilization (WHY).

In particular, there are two types of users and eleven categories of interests.

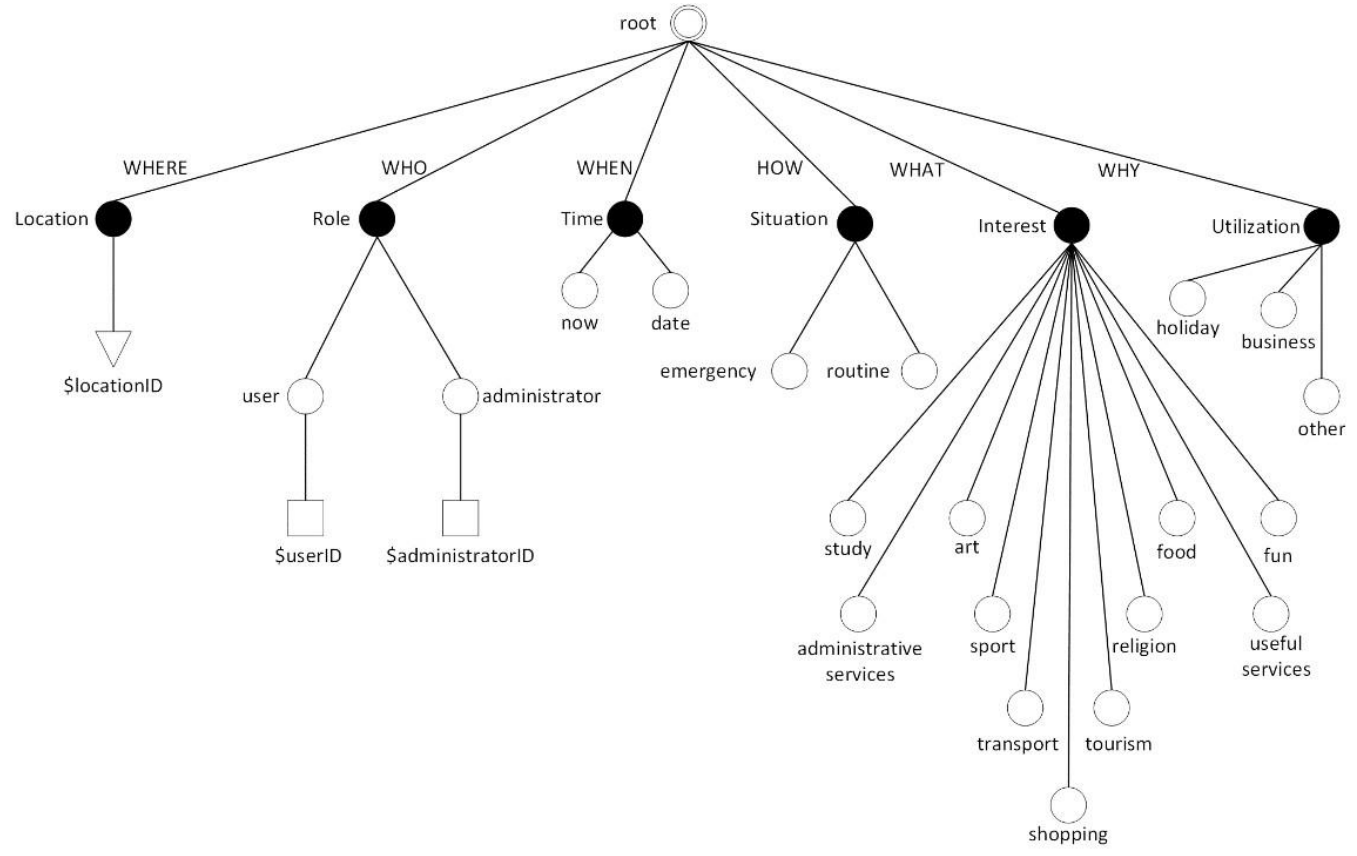

Figure 3. Meta CDT for contextual applications 
A context element is defined as an assignment d_namei = value, where d_namei indicates a possible size or undersize of CDT (it is the label of a dimension node), while value may represent the label of one of the concept nodes that are children of the considered dimension node or the value of a parameter referring to one of these concept nodes or the value of a parameter referring to the considered dimension node. For example, these assignments are possible context elements: Interest $=$ tourism, Location = LocationID $($ ID $=3)$, Role $=$ user, Utilization $=$ holiday.

A context is specified as: $\wedge\left(d_{-} n a m e i=v a l u e\right)$.

It is defined as an "and" among different context elements.

Several context elements, combined with each other by means of an "and", damage, therefore, the origin of a context.

For example, a possible framework that can be obtained from the previously seen CDT, through the context element that we have listed, is:

$$
\begin{gathered}
C=(\text { Location }=\text { locationID }(I D=3)) \wedge(\text { Role }=\text { user } \\
(I D=15)) \wedge(\text { Time }=\text { now }) \wedge(\text { Situation }=\text { routine }) \wedge \\
(\text { Interest }=\text { tourism }) \wedge(\text { Utilization }=\text { holiday })
\end{gathered}
$$

The context is defined as a user, interested in tourism, who uses the contextual app on vacation, in a called place.

Therefore, through the Context Dimension Tree, it is possible, after analyzing the domain of application, to express the size characteristics and values they can take in a graphical way by, respectively, dimension nodes and concept nodes or parameters.

The assignment to a dimension of one of its possible values is a context element. The context element can be considered the main feature of the application, by which a context can be decomposed. The moment you make the formulation of the context, you must specify all the context elements that are part of it and that enable its creation. Any context is expressible by an "and" combination of the context elements to which they are peculiar.

By definition, you can begin to understand how you will create views based on data relating to each context; in fact, they will be built starting from the portions of the database and then from the partial views, associated to the context element that takes part into context information.

The Context Dimension Tree elaboration is composed of methodologies and phases to obtain contextual service.

The methodology, shown in figure 4 , has been realized in order to manage the database and to carry out reductions of their content based on the context.

The purpose is to help the designer in the definition of all contexts relevant to the considered application and, later, in the association to each context of the portion of the database containing the relevant data about the context. The methodology consists of three main phases, which we will see in detail later: design phase of the Context Dimension Tree (CDT), definition phase of partial views and composition phase of global views.
1. Design phase of the Context Tree: in this phase, the Context Dimension Tree is designed to identify significant context elements for the considered application. In fact, it focuses on the definition of contexts and on the elements that compose them. These contexts must be identified and shaped, indicating particular elements that characterize each of them. As it has been said, it is available a special tool called Context Dimension Tree (CDT) to make context design. Three CDT were made for specific environments in order to represent and manage a multitude of different contexts and in order to identify, represent, preserve and make available cultural points for each type of user.

2. Definition phase of partial views: after the definition of all the contexts and their context elements, in this step a different portion of the database is associated to each context element, containing the relevant data for it.

In practice, the goal is to find the appropriate value for a given dimension, in order to obtain, by means of the values of all the dimensions, a valid query and specific to the context in which the user is located.

A partial view could be related to dimension "Role": once logged in, the application is able to recognize the user and to know more precisely whether he/she is, for example in tourist areas, a resident or a tourist. Thus, the value "tourist" of dimension "Role" is a partial view for the current context: using this knowledge, you can exclude certain services, not suitable or useful to the tourist role.

3. Composition phase of global views: this is the phase where you have the automatic generation of views associated with each context, which is made starting from partial views associated with context elements. After the creation of the global views of the contexts, the answers to questions that will be asked to the system will be developed from these views and, in particular, from the view associated with the context in which you are located when the query is performed.

In particular, once defined the values for each dimension, you can use all the information obtained in order to identify the right context and offer data and services customized for the user.

It is assumed the example of a tourist who is walking near a beach who gets initially a notification of his/her proximity. Later, he/she needs to deepen such notification. Therefore, it will propose him/her services that they might be interested in, such as the site of the nearest beach, where he/she can get the price list.

4.1.3. Execute phase. The third phase of the process, execute, is based on the presentation of contextualized and personalized information: therefore, the result shows itself like a well-organized story that presents a general introduction about the place reached by the user, enriched with the experiences shared by other users, a list of the main suggested attractions and information about the near places visited by the friends. 


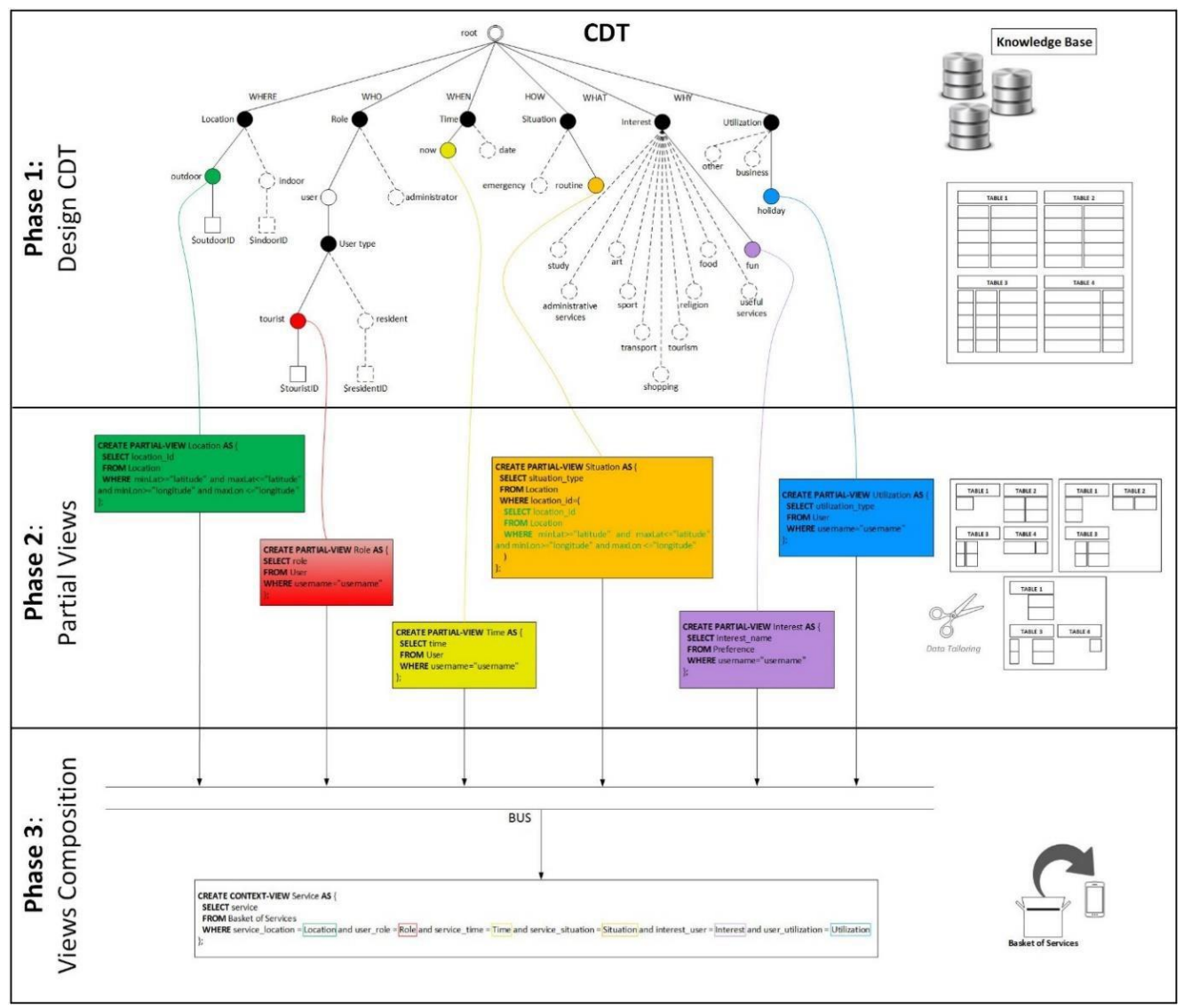

Figure 4. General System Workflow

Now, consider an example about a user called Matthew who puts some likes on social pages associated to typologies of interests: for example, school, university, library, pizza and Italian food. Each typology of interest represents a category: culture for school, university and library, restaurant for pizza and Italian food.

On the basis on this information and the data obtained on the Knowledge Base and on the web, our system determines the user's context, identifying the most appropriate resources, services and events, on the basis primarily of the profile and the user's location.

\section{An adaptive Digital Storytelling App}

In this section, we are going to present the planned and implemented app according to what previously described. The system is made available to the user through a hybrid mobile application, which allows an high level of personalization proposing a digital story that includes a series of tailor-made information, among which the suggestion of places of interest according to their current position and their own social profile.

When the user starts the app, if they do not have a Facebook account or have not logged in yet, they receive some information about the place where they are and the main points of interest that they can see on the map and reach through a navigator on the device. Once effectuated the login, the system proposes to the user a personalized story, which includes, this time, in addition to information about the place, also points of interest tailor-made suggested to the user, depending on their interests get form Facebook, and suggestions about near places that they have visited. For each of these elements it is possible to visualize the rating, the address, the distance from the current position, the preview of the users' last comments and the related web page of TripAdvisor. Furthermore, user has available contextualized services and events. Finally, if some of the user's Facebook friends have been near the current place, the system communicates when and what they have visited.

The screenshots of the application are presented in figure 5, the platform of Data Management Module is shown in figure 6. 


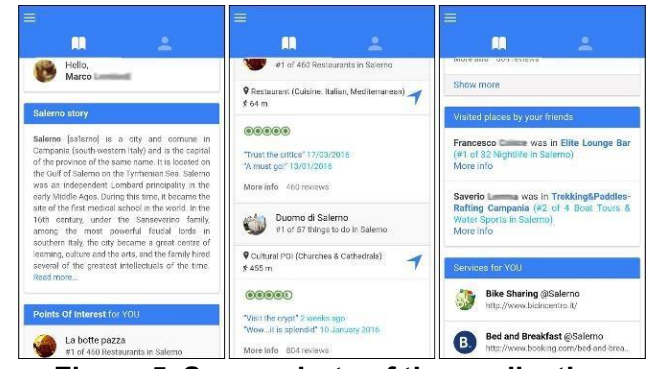

Figure 5. Screenshots of the application

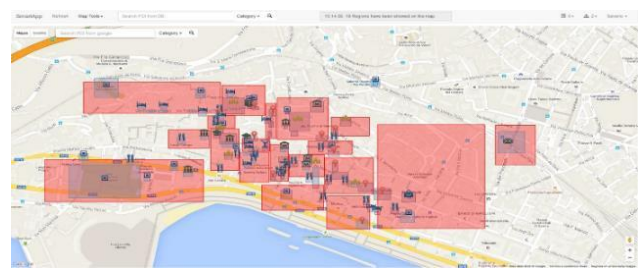

Figure 6. Platform of Data Management Module

\subsection{Experimental Results}

Now, we are going to present the conducted analysis, using the realized App, to understand the influence on the user experience of the proposed digital storytelling model.

The 40 participants, recruited by invitation, are above all students or university professors and do not know the subject of the study. All live in different towns, in Italy or abroad, are between 18 and 57 years old, are registered to Facebook and have an Android or Apple mobile device. The experimental phase is based on the storytelling model evaluation. Initially, the App has been distributed and installed by all the participants, to whom it has been requested to $\log$ in with Facebook credentials.

After having interacted for some days with the application, the participants have then answered on the basis of the Likert scale to seven statements: (1) the system gives right information about the narration of the place; (2) the system has included in my profile the categories I am mainly interested in; (3) it has proposed me points of interest and events I am mainly interested in; (4) the experience of friends and other users has been useful for me; (5) the system has suggested me tailored services; (6) the system is immediate and user-friendly; (7) the narration has been exhaustive through a personalized guide.

Table 1 presents a synthesis of the answers of the participants to each declaration.

Table 1. Experimental results

\begin{tabular}{|c|c|c|c|c|c|c|c|}
\hline Answer & $\mathbf{1}$ & $\mathbf{2}$ & $\mathbf{3}$ & $\mathbf{4}$ & $\mathbf{5}$ & $\mathbf{6}$ & $\mathbf{7}$ \\
\hline Strongly disagree & 0 & 1 & 2 & 0 & 1 & 1 & 2 \\
\hline Disagree & 2 & 3 & 5 & 2 & 3 & 2 & 2 \\
\hline Neither agree nor disagree & 5 & 4 & 5 & 3 & 9 & 6 & 4 \\
\hline Agree & 19 & 16 & 16 & 14 & 13 & 12 & 18 \\
\hline Strongly agree & 14 & 16 & 12 & 21 & 14 & 19 & 14 \\
\hline
\end{tabular}

As shown in this table, of the 40 participants who have interacted with the application, many agree and/or strongly agree that the system gives appropriate information about the place, supplies the resources of interest depending on their preferences and the final narration results complete and pertinent. Instead, only in few cases, the participants do not are particularly satisfied.

\section{Conclusions}

In this paper, it has been introduced an original approach to digital storytelling. In particular, the proposed system is based on the adaptive, social and mobile approaches, using a contextual model with the purpose to create a dynamic digital storytelling system able to collect and elaborate information and social contents about the user giving them a personalized story concerning the place they are visiting. It has been studied a real case developing a mobile application. The obtained results of the experimental campaign are satisfying and show the good potentialities of this kind of approach. The future developers can predict a greater integration of the system with new heterogeneous information sources, in order to obtain a more and more detailed and personalized final story.

\section{References}

[1] Alexander, B. and Levine, A., 2008. Web 2.0 Storytelling: Emergence of a New Genre. Educause Review, November-December 2008, 40-56, Boulder.

[2] Bae B. C., Brunete A., Malik U., Dimara E., Jermsurawong J. and Mavridis N, 2012, July. Towards an Empathizing and Adaptive Storyteller System. In Eighth Artificial Intelligence and Interactive Digital Entertainment Conference.

[3] Barthes, R. Introduction to the Structural Analysis of the Narrative. Occasional Paper, Centre for Contemporary Cultural Studies, University of Birmingham, 1966.

[4] Bedford, L., 2001. Storytelling: the real work of museums. Curator: the museum journal 44.1 (2001): 2734.

[5] Champagnat R., Delmas G., Augeraud, M., 2012. A Storytelling Model for Educational Games. Proceedings of the 6th European Conference on Games Based Learning.

[6] Colace, F., De Santo, M., Greco, L., Lemma, S., Lombardi, M., Moscato, V. and Picariello, A., 2014. Context-Aware Framework for Cultural Heritage Applications. 10th Signal-Image Technology and InternetBased Systems (SITIS).

[7] Chang, S.K., Yung, D., Colace, F., Greco, L., Lemma, S. and Lombardi, M., 2015. An Adaptive Contextual Recommender System: a Slow Intelligence Perspective. 27th Software Engineering and Knowledge Engineering (SEKE).

[8] El-Nasr, M.S., 2007. Interaction, narrative, and drama: Creating an adaptive interactive narrative using performance arts theories. Interaction Studies, 8(2), 209240.

[9] Gabriel, Y. and Connell, N.C., 2010. Co-creating stories: Collaborative experiments in storytelling. Management Learning, 41(5), 507-523.

[10] Garber-Barron, M. and Si, M., 2012, July. Towards Interest and Engagement: A Framework For Adaptive Storytelling. In Eighth Artificial Intelligence and Interactive 
Digital Entertainment Conference.

[11] Johnsson, E., 2006. Telling tales. A guide to developing effective storytelling programmes for museums. Renaissance London. C. Adler. London, London Museums Hub.

[12] Kickmeier-Rust, M.D., Göbel, S., and Albert, D., 2008. 80Days: Melding adaptive educational technology and adaptive and interactive storytelling in digital educational games. In Proceedings of the First International Workshop on Story-Telling and Educational Games (STEG'08).

[13] Ioannidis, Y., El Raheb, K., Toli, E., Katifori, A., Boile, M. and Mazura, M., 2013. One Object many stories: Introducing ICT in museums and collections through digital storytelling. Digital Heritage International Congress.

[14] Lombardo, V., and Damiano, R., 2012. Storytelling on mobile devices for cultural heritage. New Review of Hypermedia and Multimedia, 18(1-2), 11-35.

[15] Malkawi, R., 2012. Integration of multi-media technologies to facilitate reflection and learning, particularly in the area of digital storytelling. International Conference on Education and e-Learning Innovations (ICEELI).

[16] Tanca, L., Bolchini, C., Curino, C., Schreiber, F.A., 2006. Context integration for mobile data tailoring. Italian Symposium on Database Systems (SEBD), pp. 48-55.

[17] Nisi, V., Oakley, I. and Haahr, M., 2008. Location- aware multimedia stories: turning spaces into places. Universidade Catolica Portuguesa, 72-93.

[18] Paay, J., Kjeldskov, J., Christensen, A., Ibsen, A., Jensen, D., Nielsen, G. and Vutborg, R., 2008. Locationbased storytelling in the urban environment. In Proceedings of the 20th Australasian Conference on Computer-Human Interaction: Designing for Habitus and Habitat (OZCHI '08). ACM, New York, NY, USA, 122-129. DOI=http://dx.doi.org/10.1145/1517744.1517786

[19] Pearce, C., 1994. The ins \& outs of non-linear storytelling. SIGGRAPH Comput. Graph. 28, 2 (May 1994), 100-101. DOI $=10.1145 / 178951.178956$ http://doi.acm.org/10.1145/178951.178956

[20] Pittarello, F., 2006. A Simple Story: Using an Agents' Based Context-Aware Architecture for Storytelling. Technologies for interactive Digital Storytelling and Entertainment (pp 25-36).

[21] Colace, F., De Santo, M., Moscato, V., Picariello, A. Schreiber, F.A., Tanca, L., 2015e. Pervasive Systems Architecture and the Main Related Technologies. Data Management in Pervasive Systems, pp. 19-42.

[22] Colace, F., De Santo, M., Moscato, V., Picariello, A., Schreiber, F.A., Tanca, L., 2015d. PATCH: A Portable Context-Aware ATlas for Browsing Cultural Heritage.
Data Management in Pervasive Systems, pp. 345-361.

[23] Robin, B., 2006. The educational uses of digital storytelling. In C. Crawford et al. (Eds.), Proceedings of Society for Information Technology \& Teacher Education International Conference, (pp. 709-716). Chesapeake, VA: AACE.

[24] Robin, B., 2008. Digital Storytelling: A Powerful Technology Tool for the 21 st Century Classroom. Theory Into Practice, v47 n3, pp 220-228.

[25] Schumann J., Buttler T. and Lukosch S., 2010. Supporting asynchronous workspace awareness by visualizing the story evolution in collaborative storytelling. In Collaboration and Technology (pp. 218232). Springer Berlin Heidelberg. A Collaborative technology to enhance field trips.

[26] Scolari, C.A., 2009. Transmedia storytelling: Implicit consumers, narrative worlds, and branding in contemporary media production. International Journal of Communication.

[27] Spallazzo D., 2013. Davide Spallazzo - Musei, fruizione culturale e tecnologie. Storicamente, Vol. 9, ISSN:1825$411 X$.

[28] Spaniol, M., Klamma, R., Sharda, N. and Jarke M., 2006. Web-based learning with non-linear multimedia stories. In Advances in Web Based Learning-ICWL, (pp. 249-263). Springer Berlin Heidelberg.

[29] Springer, J., Borst Brazas, J. and Kajder, S., 2004. Digital storytelling at the National Gallery of Art Museums \& the Web. D. Bearman and J. Trant. Arlington, VA, Archives \& Museums Informatics.

[30] Tanenbaum, K., Hatala, M. and Tanenbaum, J., 2011. User perceptions of adaptivity in an interactive narrative. In Proceedings of the 19th international conference on User modeling, adaption, and personalization (UMAP'11), Joseph A. Konstan, Ricardo Conejo, José L. Marzo, and Nuria Oliver (Eds.). Springer-Verlag, Berlin, Heidelberg, $389-400$

[31] Colace, F., De Santo, M., Greco, L., 2014. An adaptive product configurator based on slow intelligence approach. International Journal of Metadata, Semantic and Ontologies (IJMSO), Vol.9, No.2, pp. 128-137.

[32] Colace, F., De Santo, M., Greco, L., Moscato, V. Picariello, A., 2015b. A collaborative user-centered framework for recommending items in Online Social Networks. Computers in Human Behavior 51: pp. 694- 704.

[33] Colace, F., Moscato, V., Quintarelli, E., Rabosio, E., Tanca, L., 2015c. Context awareness in pervasive information management. Data Management in Pervasive Systems, pp. 235- 256. 